\title{
A Staff Access Control System Based on RFID Technology
}

\author{
Danmei Li ${ }^{1, a^{*}}$, Huanle Yang ${ }^{2, b}$, Kayijire Fred ${ }^{3, \mathrm{c}}$, Yuxing Chen ${ }^{4, \mathrm{~d}}$ \\ ${ }^{1,2,3,4}$ College of Information Science and Technology, Donghua University, Shanghai, China \\ alidanmei@dhu.edu.cn, byang2149@163.com, cfkayijire@yahoo.fr, ${ }^{\mathrm{a}}$ yuxingcc@126.com
}

\begin{abstract}
Keywords: RFID, Access Control System, Work Attendance.
Abstract. A staff access control system based on RFID technology is designed in this paper. This staff access control system is comprised of UHF RFID readers, industrial personal computers, a database server, big screen monitors, and RFID handsets. It is mostly applied to the main entrances of an organization outside a building. The system identifies people based on a match between the personnel's tag ID stored in the tag and the tag ID records stored in the database. Each time someone with an available tag passes a gate, a staff access record is added into the database. The accessing staffs' identification information is displayed on a big screen in real time. With a little bit of searching work, any staff's work attendance information can be obtained. With all these features, the system is capable of doing access management and check on work attendance management at the same time.
\end{abstract}

\section{Introduction}

With the development of internet technology and the wide spread of RFID technology, the access control systems have been applied to diverse organizations. They can be applied to inner side of a building, such as meeting rooms and laboratories. They can also be applied to the outside of a building, such as main entrances of research organizations and government buildings. Some researches on the laboratories access control systems have been done[1,2] in the few pass years. A laboratory access control system is comprised of electromagnetic locks, RFID readers, exit switches, an ARM chip micro-controller[3] and RFID tags.

This paper designed a staff access control system applied to the outside of a building, where visitors traffic can be very high. For information security reasons, organizations always isolate the intranet from the internet. This system runs on the intranet. In order to gather data and store data, RFID technology and database is applied in this system. Records of access information can be filtered to get the information of staff work attendance[4]. This system gathers data with RFID readers, identifies people with staff recognition processor, stores staff information and access information with database.

\section{RFID Technology Theorem}

RFID (Radio Frequency Identification) technology is a kind of technology which is able to identify active and passive tags within a range of a few meters. The theorem is shown in Fig. 1.

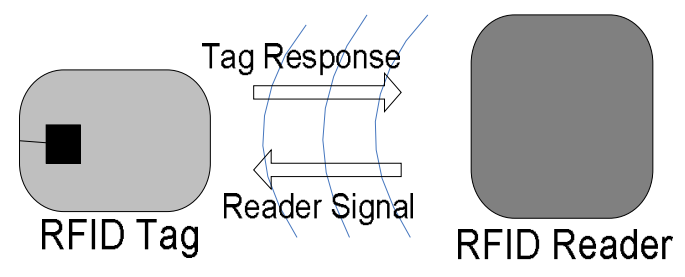

Fig. 1. RFID Technology Theorem

The inner components of RFID tags are a coil and a micro-chip which has a little storage capability. The appearance of RFID tags are diverse. The coil and chip can be encapsulated into label paper, round plastic coins or plastic tubes. The RFID reader sends out some specific frequency of electromagnetic wave. The wave carries a signal. When there are RFID tags within the range of the a reader's working distance, the tags induce the signal, and reflect with electromagnetic wave of corresponding frequency . 
This wave is the response signal of tags. When the RFID reader receives the signal, the data stored in the tag is obtained.

Although RFID technology has been put into use since World War II, the real wide spread of this technology is after that the America's biggest retailer Wal-Mart mandated its one hundred suppliers to apply this technology[5]. RFID tags used in this system is read-only. Tag ID that stored in the micro-chip is an array of hexadecimal numbers. An example is shown in table 1.

\begin{tabular}{cccccccccccccc}
\multicolumn{1}{c}{ Table 1 An Example of Tag ID That Stored in The Micro-chip } \\
\hline Byte & 1 & 2 & 3 & 4 & 5 & 6 & 7 & 8 & 9 & 10 & 11 & 12 \\
\hline value & 10 & 00 & 21 & 00 & 01 & 06 & $0 \mathrm{~A}$ & 03 & $3 \mathrm{E}$ & 00 & $1 \mathrm{E}$ & $0 \mathrm{~A}$ \\
\hline
\end{tabular}

Every staff in an organization is issued with a tag. The tag ID is stored in the database corresponding to the staff's identification information, such as the name, the department, the picture, and so on.

\section{System Architecture}

The staff access control system designed in this paper runs on the intranet of the organization. The intranet is completely isolated from the internet. The architecture of the system is shown in Fig. 2.

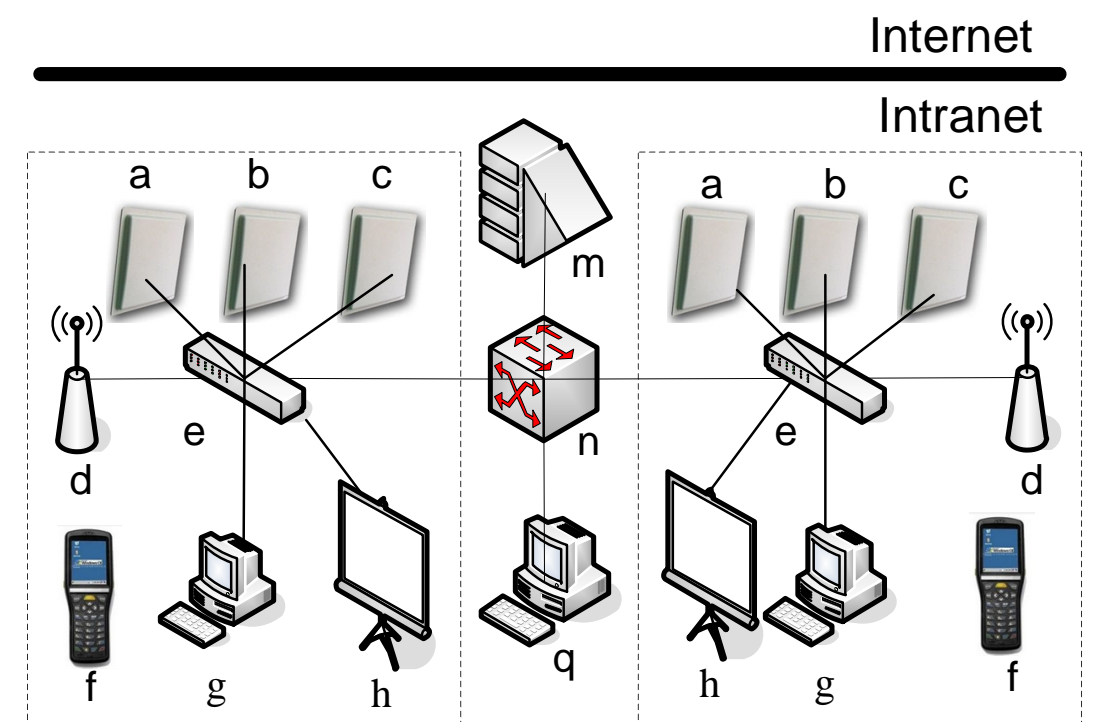

Fig. 2. System Architecture. a) RFID reader b) RFID reader c) RFID reader d) wireless access point e) switch f) RFID handset $\mathrm{g}$ ) staff access recognition processor $\mathrm{h}$ ) staff access monitor $\mathrm{m}$ ) database server $\mathrm{n}$ ) router $\mathrm{q}$ ) inquiry computer

The access control system is comprised of multiple subsystems. Each subsystem is installed at each gate of the organization. The subsystems are identical. The subsystem is comprised of three RFID readers, one staff access identification processor, a staff access status monitor, a wireless access point, and several handsets. The devices in the subsystem are connected to the intranet via a switch. Any computer in the intranet can be used as an inquiry computer, which is capable of accessing the staff information stored in database server.

\section{System Functionality}

The access control system designed in this paper provides functions such as staff identification, staff information management, staff access records storage, staff RFID tags information management and staff work attendance management. The handsets in the system are able to read RFID tags, and display 
the staff information. When a staff who carries a tag passes through a gate, a RFID reader reads the tag. The staff access identification processor compares the tag ID with the records in the database to determine the availability of the tag. If the tag is available, the access information will be recorded, and the staff's identification information will be displayed. If the tag is not available, or there is no tag, the system will alert. Then security staff will use the handset to confirm again. When there are personnel changes, the staff information can be updated with the system maintenance. When someone's RFID tag was lost or unavailable, he or she will be issued with a new tag, and the staff RFID tags information needs to be updated. Through a little searching work on the access records, the organization could get the workers' attendance information.

\section{RFID Reader Selection}

There are two models of RFID readers being taken into consideration, Shenzhen AFD RFID technology Co., Ltd AFD-8580 RFID reader and AWID company MPR-2010BN RFID reader. Both of their working frequency is $902 \sim 928 \mathrm{MHz}$.

Table 2 AFD-8580 RFID Reader and MPR-2010BN RFID Reader Parameters

\begin{tabular}{cccccc}
\hline & Model & SRD $(\mathrm{m})$ & Standards & M TRA(tags) & TRR(tags/s) \\
\hline 1 & AFD-8580 & $12 \sim 15$ & ISO18000-6B/C & 300 & 300 \\
2 & MPR-2010BN & $5-6$ & ISO18000-6B/C & & \\
\hline
\end{tabular}

In table 2, SRD stands for Steady Reading Distance, MTRA stands for Multiple Tag Reading Ability, TRR stands for Tag Reading Rate. Although the multiple tag reading ability and tag reading rate of model MPR-2010BN is not provided, the two parameters is much less than these of AFD-8580 from field test. In addition, the AFD-8580 model is better than the MPR-2010BN model in stability. Therefore the choice would be the AFD-8580 model.

\section{Database}

For organizations with thousands of workers and multiple departments, the access control system provides a SQL server database to supply data services, as shown in Fig. 3.

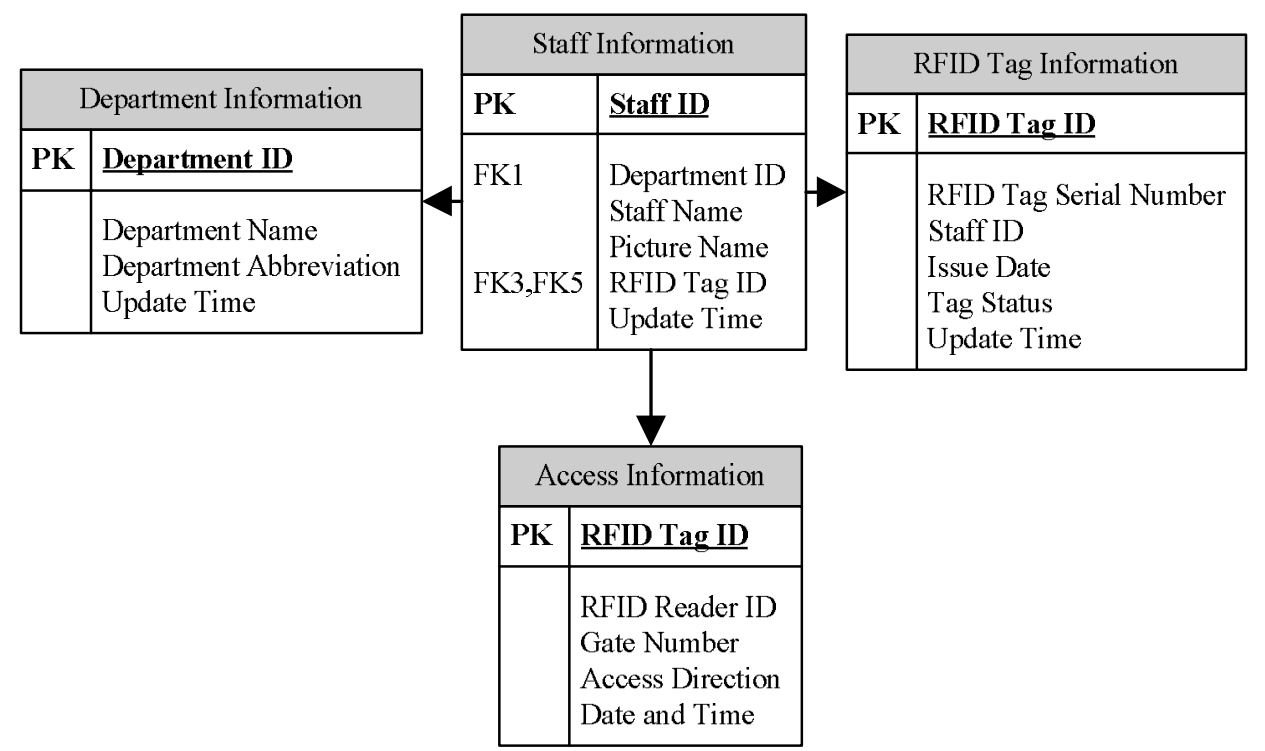

Fig. 3. Table Structures of Database 
There are four tables in the database. Each of them records the staff information, the department information, the RFID tag information and the access information. The staff information includes unit ID, staff name, picture name, RFID tag ID, update time. The department information includes department ID, department name, department abbreviation and update time. The RFID tag information includes RFID tag ID, RFID tag serial number, staff ID, issue date, tag status and update time. The access information includes RFID tag ID, gate number, access direction and time. When there are personnel changes, the department information table and staff information table should be updated. When someone lost a tag, when he or she is issued with a new tag, the RFID tag information table should be updated too.

\section{Software Design}

In order to improve the stability of the whole system, the software of the access control system runs on the staff recognition processors which are industrial computers. The industrial computers are located at the same local area network with the database server. The flow-process diagram of the program is shown in Fig. 4.

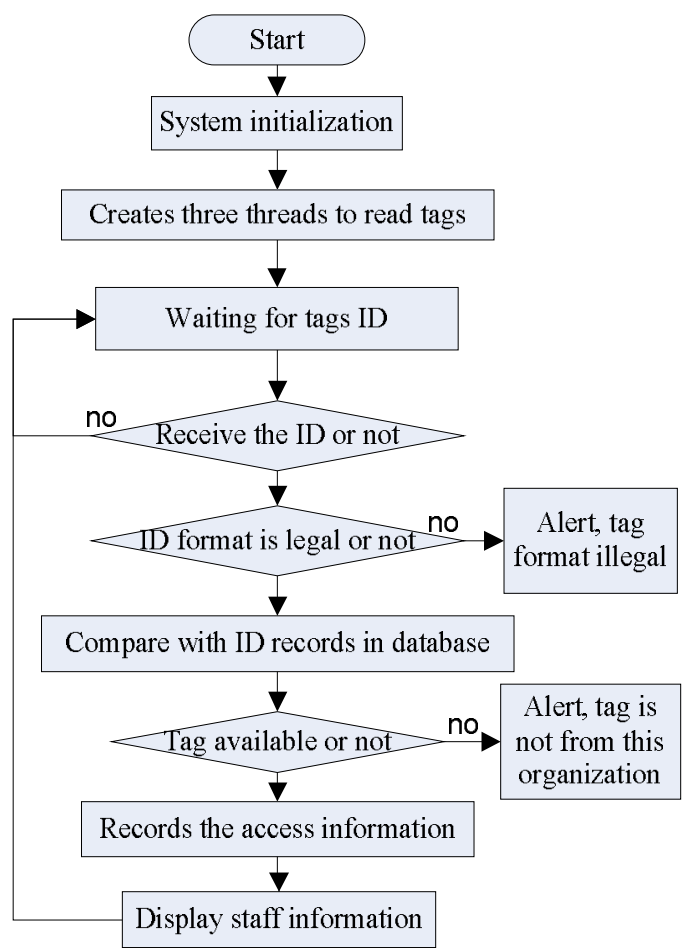

Fig. 4. Program Flow-process Diagram

When the access control system starts, it does some initialization work. The initialization work includes initial variables of the program, tests the parameters of the RFID readers, and so on. Then three tag reading threads are created corresponding to three RFID readers. The RFID readers send ultra high frequency electromagnetic wave, and receive the RFID tag ID. The readers are able to receive multiple tag IDs at the same time.

When the tag ID is received, it first test if the ID format is legal. The system will alert if the format is illegal. Next, the ID will be compared with the ID records in the database. If the tag is available, there are two things need to be done. First, the record will be stored. Second, the RFID tag owner's information will be displayed on the monitor, such as the department, the name, the time and the picture. If the tag is not available, the system will alert the security staff that there are non-staff people try to get in. 


\section{RFID Reader Test}

The RFID reader test is to research about two problems. The first problem is to compare the reading rate between the response mode and the active mode to find out which is faster. The second one is to find out the interference between two readers if they are nearby. The test results are shown in table 3 and table 4. Two readers are placed in parallel with working side point to the same direction. The distance between two readers is about one meter. All three tags are placed on the central line away from the readers about two meters.

Table 3 shows that the frequency of each tag read by one reader in response mode in one minute is approximately equal to that in active mode. Table 4 shows that the sum of frequency of each tag read by two readers in response mode in one minute is approximately equal to that in active mode. In table 4 , the tag 1a means the tag 1 is read by the first RFID reader, tag $1 \mathrm{~b}$ means the tag 1 is read by the second RFID reader, and so on.

Table 3 Response and Active Mode with One Reader in One Minute Test

\begin{tabular}{cccc}
\hline Tag Number & tag1 & tag2 & tag3 \\
\hline Frequency in Response Mode & 919 & 932 & 915 \\
Frequency in Active Mode & 985 & 1001 & 1008 \\
\hline
\end{tabular}

Table 4 Response and Active Mode with Two Readers in One Minute Test

\begin{tabular}{ccccccc}
\hline Tag Number & tagla & tag2a & tag3a & tag1b & tag2b & tag3b \\
\hline Frequency in Response Mode & 775 & 445 & 681 & 139 & 512 & 263 \\
Frequency in Active Mode & 398 & 510 & 739 & 618 & 537 & 338 \\
\hline
\end{tabular}

The sum of number of each tag read in response mode is a little bit less than that in active mode. So the reading rate of the response mode and active mode is almost the same. The frequency of tags read by two readers are distinct, and they are also different from those read only by one reader. These features prove that the readers are fighting against each other for the signal when they are reading a tag at the same time. A tag can only deal with one reader at a time. The interference does exist when two or more readers working together nearby. To avoid this problem, necessary considerations must be taken when installing RFID readers.

\section{System Test}

The configuration of the database server, industrial computers and RFID readers within the same local area network should be tested to rule out any communication problems. In the first test, the system is able to work, but several problems occurred. The reading rate is too slow. Some tags are missed when dozens of tags are read together. The software can't connect to server when the server reboots. New problems should be fixed. As for the low reading rate, new threads are created in the program to read tag ID exclusively. When the threads get the tag ID, it passes the ID to the next part of the program, and go back to read next ID again. As for the missing tags problem, the ISO18000-6C protocol is applied to enable the reader to read hundreds of tags at the same time. To get around the connection errors, an option on the socket should be set before bind to a port in the program. When all problems are fixed, the system runs as demanded. The access status information is shown in Fig.5. 


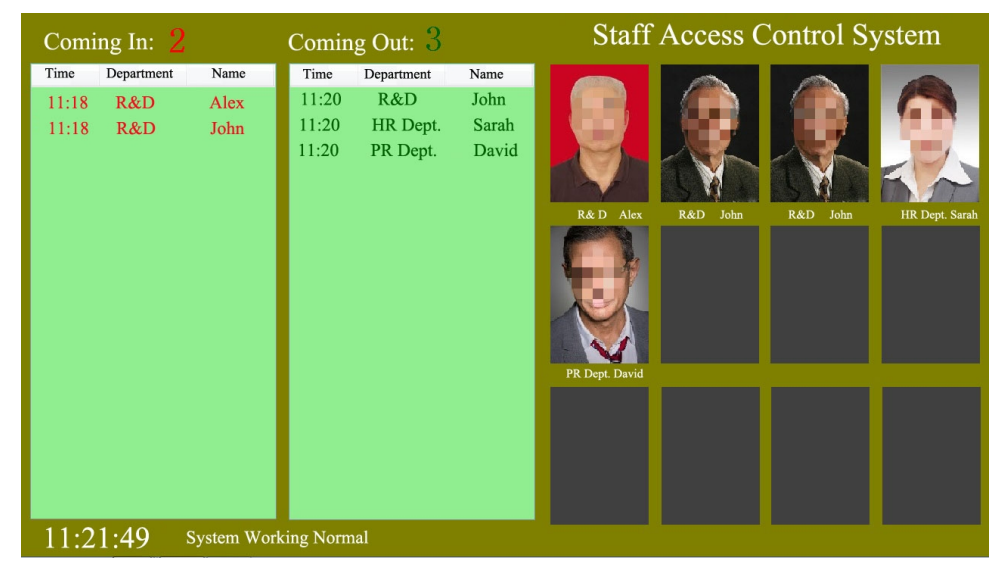

Fig. 5. Access Status Information

\section{Conclusions}

In order to deal with the heavy traffic problem in the main entrances of a big organization, a staff access control system based on RFID technology is designed in this paper. This system runs on the intranet of the organization. The intranet is isolated from the internet, so that the information security of the system is guaranteed. The AFD-8580 UHF RFID readers in the system provide enough reading distance ability, multiple tags reading ability, good reading speed, and stability. It is able to deal with crowded situations. In order to provide enough process ability and stability, professional industrial computers are applied as the staff identification processors. The database is used to record staff access information, update staff information and RFID tag information. The work attendance information can be searched from the database. All of these features provide a convenient tool to manage staff access and check on work attendance.

\section{References}

[1] Yahui Wen, The design and implementation of access system based on RFID, J. Information Communication. 2012, 6(61), 61, 221. In Chinese.

[2] Wanliang Zhang, The design of laboratory access system based on RFID, J. Design and Development. 2013, 22(16), 16-17, 20. In Chinese.

[3] Aiping Wu, Chao Xiong, Jun Wei, Wei Tang, Tianfa Yang, The design of RFID access control system based on ARM, J. Modern Electronic Technology. 2013, 36(2), 60-61, 67. In Chinese.

[4] Shengnan $\mathrm{Hu}$, Zhouping Yin, Xubing chen. The design and implementation based on RFID access and work attendance system, J. Digital Technology and application. 2010, (9), 76-79. In Chinese.

[5] Yu-Chih Huang, Secure Access Control Scheme of RFID System Application, J. $5^{\text {th }}$ International Conference on Information Assurance and Security. 2009, (1), 525-528.

[6] G. Roussos, Networked RFID, Systems, Software and Services, first ed., University of London, UK, 2008. 